\title{
Bayes Estimation of the Mean of Normal Distribution Using Moving Extreme Ranked Set Sampling
}

\author{
Said Ali Al-Hadhrami \\ College of Applied Science \\ Nizwa, Oman \\ abur1972@yahoo.co.uk \\ Amer lbrahim Al-Omari \\ Department of Mathematics \\ Al al-Bayt University, Mafraq, Jordan \\ alomari_amer@yahoo.com
}

\begin{abstract}
Moving extreme ranked set sampling(MERSS)(Al-Saleh and Al-Hadrami $2003 \mathrm{a}, \mathrm{b}$ ) is one of useful modifications of the usualranked setsampling(RSS).This method uses only extremes ranks with varied set size to reduce error in ranking. In this paper, Bayes estimation of the mean of normal distribution based on MERSSwas considered and compared with simple random sampling (SRS). counterpart. The suggested estimatorsare found to be more efficient than that from SRS.
\end{abstract}

Keywords: Moving extreme ranked set sampling, Bayes estimator, Efficiency.

\section{Introduction}

There are some studies investigated ranked set sampling (RSS) from a Bayesian point of view. Al-Saleh and Muttlak (1998) investigated Bayesian estimators of the mean of the exponential distribution. Lavine (1999) studied some aspects of Bayesian RSS. Kim and Arnold (1999) considered Bayesian estimation under both balanced and generalized RSS. Al-Saleh and Muttlak (2000) considered Bayesian estimation using RSS and found a Bayes estimator of exponential distribution under conjugate prior and gave an application of real data. AlSalehand Abu Hawwas (2002) used the notion of multiple imputations to characterize the Bayes estimators using RSS. This characterization was used to approximate complicated Bayesian estimators and was applied to the case of normal distribution with conjugate prior. For more details about RSS see Koyuncu and Kadilar (2009), Li and Balakrishnan (2008), Al-Omari and Jaber (2008), and Al-Omari, et al. (2009).

Moving extreme ranked set sampling was proposed by Al-Odat and Al-Saleh (2001). This method uses only extremes with varied set size to reduce error in ranking. Al-Saleh and Al-Hadhrami (2003a,b) investigated the method parametrically and found maximum likelihood estimators (MLE) of some parameters of normal, exponential and uniform distributions. Based on MERSS, several ratio, product and chain type estimators were studied by Al-Hadhrami (2007). Abu-Dayyeh and Al-Sawi (2007) investigated inference about the mean of the exponential distribution using MERSS. Estimation of the mean using 
concomitant variable was studied by Al-Saleh and Al-Ananbeh (2007). AlHadhrami et al. (2009) found the MLE of variance of the normal distribution and investigated its properties. This MLE is unbiased estimator and more efficient than the competitor estimator from SRS. Bayesian inference on the variance of normal distribution using MERSS was considered by Al-Hadhrami and Al-Omari (2010). Generalized MLE, confidence intervals, and different testing hypotheses were considered. It was shown that the modified inferences using MERSS are more efficient than their counterparts based on SRS. Al-Saleh and Samawi (2010) considered the estimation of the odds, F/(1 - F) based on MERSS, and showed that the estimator based on MERSS have some advantages over that based on SRS.

In this paper, Bayesian estimator of the population mean of normal distribution is considered and compared with estimators based on simple random sampling.

The remaining part of this paper is organized as follows: Bayes estimator of the mean based on MERSS is given in Section 2. In Section 3, Bayes estimators of the mean of normal distribution are considered based on constant and conjugate priors. An approximation of $\hat{\theta}_{\mathrm{MERSS}}$ is given in Section 4. In Section 5 a simulation study is presented to evaluate the performance of the suggested estimators. Finally, conclusions are given in Section 6.

\section{Bayes estimator of the mean based on MERSS}

The MERSS can be summarized as follows:

Step 1: Select $m$ random samples of size $1,2,3, \ldots, m$, respectively.

Step 2: Identify the maximum of each set, visually or by any cost free method without actual measurement of the variable of interest.

Step 3: Measure accurately the selected judgment identified maxima.

Step 4: Repeat Steps 1, 2, 3 but for minimum of each set.

Step 5: Repeat the above steps $r$ times until the desired sample size, $n=2 r m$ is obtained.

For one cycle, the following MERSS sample is obtained

$$
X_{m: m}, X_{m-1: m-1}, X_{m-2: m-2}, \ldots, X_{1: 1}, X_{1: m}, X_{1: m-1}, X_{1: m-2}, \ldots, X_{1: 1} \text {, }
$$

where $X_{i: j}$ is the element of rank $i$ from a set of size j. For simplicity, use $X_{i}$ for $X_{i: i}$ and $Y_{j}$ for $X_{1: j}$. Then, the sample of one cycle is given by

$$
X_{1}, X_{2}, X_{3}, \ldots, X_{m}, Y_{1}, Y_{2}, Y_{3} \ldots, Y_{m}
$$

The notion of multiple imputations used by Al-Salehand Abu Hawwas (2002) can be also applied for MERSS with similar formula developed for RSS. Assume that $X_{1}^{*}, X_{2}^{*}, \ldots, X_{2 m}^{*}$ to be a SRS with density $f\left(X^{*} \mid \theta\right)$ and cumulative distribution function $F\left(X^{*} \mid \theta\right)$. Let $X_{1}, X_{2}, \ldots, X_{m}, Y_{1}, Y_{2}, L, Y_{m}$ be a MERSS of one cycle from this distribution obtained using a full data of $m(m+1)$ observations. 
Let () be a prior density of and $\pi(\theta \mid x, y)$ be the posterior density of $\theta$ given $\mathrm{x}, \mathrm{y}$ and $\pi\left(\theta \mid \mathrm{x}^{*}\right)$ is the posterior density of $\theta$ given $\mathrm{x}^{*}$. Now, let us introduce the following identity based on MERSS that is similar to one provided by Rubin (1978).

$$
\pi(\theta \mid \mathrm{x}, \mathrm{y})=\int_{\mathrm{x}^{*}} \pi\left(\theta \mid \mathrm{x}^{*}\right) \mathrm{m}\left(\mathrm{x}^{*} \mid \mathrm{x}, \mathrm{y}\right) \mathrm{dx}
$$

where

and

$$
m\left(x^{*} \mid x, y\right)=\frac{m\left(x^{*}, x, y\right)}{m(x, y)}, m\left(x^{*}, x, y\right)=\int_{-\infty}^{\infty} \pi(\theta) f\left(x^{*}, x, y \mid \theta\right) d \theta
$$

$$
m(x, y)=\int_{x^{*}} m\left(x^{*}, x, y\right) d x^{*}
$$

Based on this identity, the posterior density of $\theta$ given a MERSS data of size $2 \mathrm{~m}$ is the expected value of the posterior density of $\theta$, given the SRS data of size $m(m+1)$, i. e., full data. The expectation is with respect to the predictive distribution, $m\left(x^{*} \mid x, y\right)$.

Bayes estimator of $\theta$ under the squared error loss function (SELF) is the mean of the posterior density. Therefore, from (1) we have

$$
\int_{\theta} \theta \pi(\theta \mid \mathrm{x}, \mathrm{y}) \mathrm{d} \theta=\int_{\theta} \theta\left[\int_{x^{*}} \pi\left(\theta \mid \mathrm{x}^{*}\right) \mathrm{m}\left(\mathrm{x}^{*} \mid \mathrm{x}, \mathrm{y}\right) \mathrm{dx}\right] \mathrm{d} \theta,
$$

and

$$
\hat{\theta}_{\text {MERSS }}=\int_{x^{*}} \mathrm{~m}\left(\mathrm{x}^{*} \mid \mathrm{x}, \mathrm{y}\right)\left[\int_{\theta} \theta \pi\left(\theta \mid \mathrm{x}^{*}\right) \mathrm{d} \theta\right] \mathrm{d} \mathrm{x}^{*}=\int_{\mathrm{x}^{*}} \hat{\theta}_{\mathrm{SRS}} \mathrm{m}\left(\mathrm{x}^{*} \mid \mathrm{x}, \mathrm{y}\right) \mathrm{dx} \mathrm{x}^{*},
$$

where $\hat{\theta}_{\mathrm{MERSS}}$ and $\hat{\theta}_{\mathrm{SRS}}$ are the Bayes estimators of $\theta$ using MERSS and SRS methods, respectively.

Formula (2) shows that the MERSS Bayes estimator is the expected value of the SRS Bayes estimator based on the full data with respect to the predictive distribution.

From the relation

we got

$$
\operatorname{Var}(\theta \mid \mathrm{x}, \mathrm{y})=\mathrm{E}\left[\operatorname{Var}\left(\theta \mid \mathrm{x}^{*}\right) \mid \mathrm{x}, \mathrm{y}\right]+\operatorname{Var}\left[\mathrm{E}\left(\theta \mid \mathrm{x}^{*}\right) \mid \mathrm{x}, \mathrm{y}\right]
$$

$$
\operatorname{Var}(\theta \mid \mathrm{x}, \mathrm{y})=\mathrm{E}\left[\operatorname{Var}\left(\theta \mid \mathrm{x}^{*}\right) \mid \mathrm{x}, \mathrm{y}\right]+\operatorname{Var}\left[\hat{\theta}_{\text {SRS }}\left(\mathrm{x}^{*}\right) \mid \mathrm{x}, \mathrm{y}\right] \text {. }
$$

The Bayes risk of $\hat{\theta}_{\mathrm{MERSS}}$ is

$$
r\left(\hat{\theta}_{\mathrm{MERSS}}(\mathrm{x}, \mathrm{y}), \pi\right)=\mathrm{E}_{\theta} \mathrm{E}_{\mathrm{x}, \mathrm{y}}\left[\left(\hat{\theta}_{\mathrm{MERSS}}-\theta\right)^{2}\right] \text {, }
$$

and the Bayes risk of $\hat{\theta}_{\mathrm{SRS}}$ is 
Said Ali Al-Hadhrami, Amer Ibrahim Al-Omari

$$
r\left(\hat{\theta}_{\text {SRS }}\left(x^{*}\right), \pi\right)=\mathrm{E}_{\theta} \mathrm{E}_{x^{*}}\left[\left(\hat{\theta}_{\text {SRS }}-\theta\right)^{2}\right] .
$$

Then, using (3), we obtain

$$
\begin{aligned}
& r\left(\hat{\theta}_{\text {MERSS }}(\mathrm{x}, \mathrm{y}), \pi\right)=\mathrm{E}\left\{\mathrm{E}\left[\operatorname{Var}\left(\theta \mid \mathrm{x}^{*}\right) \mid \mathrm{x}, \mathrm{y}\right]\right\}+\mathrm{E}\left\{\operatorname{Var}\left[\hat{\theta}_{\text {SRS }}^{*}\left(\mathrm{x}^{*}\right) \mid \mathrm{x}, \mathrm{y}\right]\right\} \\
= & \mathrm{r}\left(\hat{\theta}_{\text {SRS }}\left(\mathrm{x}^{*}\right), \pi\right)+\mathrm{E}\left[\operatorname{Var}\left(\hat{\theta}_{\text {SRS }}\left(\mathrm{x}^{*} \mid \mathrm{x}, \mathrm{y}\right)\right)\right] .
\end{aligned}
$$

\section{Bayes estimator of the mean of normal distribution}

Assume that $\mathrm{f}$ is the density of a normal random variable with mean $\theta$ and variance $1, X \sim N(\theta, 1)$. Our interest is to get Bayes estimate of $\theta$ based on MERSS. For simplicity, we use $X_{i}, Y_{i}$ for $X_{i: i}$ and $Y_{1: i}$, respectively. The joint density function of $X$ and $Y$ is

$$
\mathrm{f}(\mathrm{x}, \mathrm{y} ; \theta)=\prod_{\mathrm{i}=1}^{\mathrm{m}} \mathrm{i}^{2} \mathrm{f}\left(\mathrm{x}_{\mathrm{i}}, \theta\right)\left[\mathrm{F}\left(\mathrm{x}_{\mathrm{i}}, \theta\right)\right]^{\mathrm{i}-1} \mathrm{f}\left(\mathrm{y}_{\mathrm{i}}, \theta\right)\left[1-\mathrm{F}\left(\mathrm{y}_{\mathrm{i}}, \theta\right)\right]^{\mathrm{i}-1} \text {. }
$$

Substitute $\mathrm{f}(\mathrm{x}, \theta)=\phi(\mathrm{x}-\theta)$ and $\mathrm{F}(\mathrm{x}, \theta)=\Phi(\mathrm{x}-\theta)$ in (5), we get

$$
\mathrm{f}(\mathrm{x}, \mathrm{y} ; \theta)=\prod_{\mathrm{i}=1}^{\mathrm{m}} \mathrm{i}^{2} \phi\left(\mathrm{x}_{\mathrm{i}}-\theta\right)\left[\Phi\left(\mathrm{x}_{\mathrm{i}}-\theta\right)\right]^{\mathrm{i}-1} \phi\left(\mathrm{y}_{\mathrm{i}}-\theta\right)\left[1-\Phi\left(\mathrm{y}_{\mathrm{i}}-\theta\right)\right]^{\mathrm{i}-1} \text {. }
$$

Now, suppose that $\theta \sim h(\theta)$ as a prior density. Thus, the posterior density is given by

$$
\mathrm{h}(\theta ; \mathrm{x}, \mathrm{y})=\frac{\mathrm{h}(\theta) \prod_{\mathrm{i}=1}^{\mathrm{m}} \mathrm{i}^{2} \phi\left(\mathrm{x}_{\mathrm{i}}-\theta\right)\left[\Phi\left(\mathrm{x}_{\mathrm{i}}-\theta\right)\right]^{\mathrm{i}-1} \phi\left(\mathrm{y}_{\mathrm{i}}-\theta\right)\left[1-\Phi\left(\mathrm{y}_{\mathrm{i}}-\theta\right)\right]^{\mathrm{i}-1}}{\int_{-\infty}^{\infty} \mathrm{h}(\theta) \prod_{\mathrm{i}=1}^{\mathrm{m}} \mathrm{i}^{2} \phi\left(\mathrm{x}_{\mathrm{i}}-\theta\right)\left[\Phi\left(\mathrm{x}_{\mathrm{i}}-\theta\right)\right]^{\mathrm{i}-1} \phi\left(\mathrm{y}_{\mathrm{i}}-\theta\right)\left[1-\Phi\left(\mathrm{y}_{\mathrm{i}}-\theta\right)\right]^{\mathrm{i}-1} \mathrm{~d} \theta}
$$

The Bayes estimator using square error loss function, which is the mean of posterior distribution is

$$
\hat{\theta}=\frac{\int_{-\infty}^{\infty} \theta \cdot \mathrm{h}(\theta) \prod_{\mathrm{i}=1}^{\mathrm{m}} \mathrm{i}^{2} \phi\left(\mathrm{x}_{\mathrm{i}}-\theta\right)\left[\Phi\left(\mathrm{x}_{\mathrm{i}}-\theta\right)\right]^{\mathrm{i}-1} \phi\left(\mathrm{y}_{\mathrm{i}}-\theta\right)\left[1-\Phi\left(\mathrm{y}_{\mathrm{i}}-\theta\right)\right]^{\mathrm{i}-1} \mathrm{~d} \theta}{\int_{-\infty}^{\infty} \mathrm{h}(\theta) \prod_{\mathrm{i}=1}^{m} \mathrm{i}^{2} \phi\left(\mathrm{x}_{\mathrm{i}}-\theta\right)\left[\Phi\left(\mathrm{x}_{\mathrm{i}}-\theta\right)\right]^{\mathrm{i}-1} \phi\left(\mathrm{y}_{\mathrm{i}}-\theta\right)\left[1-\Phi\left(\mathrm{y}_{\mathrm{i}}-\theta\right)\right]^{\mathrm{i}-1} \mathrm{~d} \theta} .
$$

In the following we will introduce the Bayes estimators of the $\theta$ based on constant and conjugate priors.

3.1 Bayes estimator of the mean of normal distribution with constant prior Assuming that $\pi(\theta)=1$, then the Bayes estimator of $\theta$ is

$$
\hat{\theta}_{\mathrm{MERSS}}^{\delta}=\frac{\int_{\theta} \theta \prod_{\mathrm{i}=1}^{\mathrm{m}} \phi\left(\mathrm{x}_{\mathrm{i}}-\theta\right) \Phi^{\mathrm{i}-1}\left(\mathrm{x}_{\mathrm{i}}-\theta\right) \phi\left(\mathrm{y}_{\mathrm{i}}-\theta\right)\left[1-\Phi\left(\mathrm{y}_{\mathrm{i}}-\theta\right)\right]^{\mathrm{i}-1} \mathrm{~d} \theta}{\int \prod_{\theta}^{m} \phi\left(\mathrm{x}_{\mathrm{i}}-\theta\right) \Phi^{\mathrm{i}-1}\left(\mathrm{x}_{\mathrm{i}}-\theta\right) \phi\left(\mathrm{y}_{\mathrm{i}}-\theta\right)\left[1-\Phi\left(\mathrm{y}_{\mathrm{i}}-\theta\right)\right]^{\mathrm{i}-1} \mathrm{~d} \theta} .
$$

The following lemma summarizes some properties of this estimator. 
Bayes Estimation of the Mean of Normal Distribution Using Moving Extreme Ranked Set Sampling

Lemma 1: For any scalar "a", and $a=(a, a, \ldots . ., a)$, we have

1. $\hat{\theta}_{\text {MERSS }}^{\delta}(\mathrm{x}+\mathrm{a}, \mathrm{y}+\mathrm{a})=\hat{\theta}_{\mathrm{MERSS}}^{\delta}(\mathrm{x}, \mathrm{y})+\mathrm{a}$.

2. $\hat{\theta}_{\text {MERSS }}^{\delta}(-(\mathrm{x}, \mathrm{y}))=-\hat{\theta}_{\mathrm{MERSS}}^{\delta}(\mathrm{x}, \mathrm{y})$.

3. $\hat{\theta}_{\mathrm{MERSS}}^{\delta}$ is an unbiased estimator of $\theta$.

4. The risk function of $\hat{\theta}_{\mathrm{MERSS}}^{\delta}$ is free of $\theta$.

Proof:

1. Consider the sample $x+a, y+a$. Then, the estimator based on these observations is given by

$$
\begin{aligned}
& \hat{\theta}_{\text {MERSS }}^{\delta}=\frac{\int_{\theta}(\theta+a-a) \prod_{i=1}^{m} \phi\left(x_{i}+a-\theta\right) \Phi^{i-1}\left(x_{i}+a-\theta\right) \phi\left(y_{i}+a-\theta\right)\left[1-\Phi\left(y_{i}+a-\theta\right)\right]^{i-1} d \theta}{\int \prod_{i=1}^{m} \phi\left(x_{i}+a-\theta\right) \Phi^{i-1}\left(x_{i}+a-\theta\right) \phi\left(y_{i}+a-\theta\right)\left[1-\Phi\left(y_{i}+a-\theta\right)\right]^{i-1} d \theta} \\
= & \frac{\int_{\theta}(\theta-a) \prod_{i=1}^{m} \phi\left(x_{i}-(\theta-a)\right) \Phi^{i-1}\left(x_{i}-(\theta-a)\right) \phi\left(y_{i}-(\theta-a)\right)\left[1-\Phi\left(y_{i}-(\theta-a)\right)\right]^{i-1} d \theta}{\int_{\theta} \prod_{i=1}^{m} \phi\left(x_{i}-(\theta-a)\right) \Phi^{i-1}\left(x_{i}-(\theta-a)\right) \phi\left(y_{i}-(\theta-a)\right)\left[1-\Phi\left(y_{i}-(\theta-a)\right)\right]^{i-1} d \theta}+a \\
\therefore & \hat{\theta}_{\text {MERSS }}^{\delta}(x+a, y+a)=\hat{\theta}_{\text {MERSS }}^{\delta}(x, y)+a .
\end{aligned}
$$

2. Consider the sample $-x,-y$. Then, the estimator based on these observations is

$$
\begin{gathered}
\hat{\theta}_{\text {MERSS }}^{\delta}=\frac{\int_{\theta} \theta \prod_{\mathrm{i}=1}^{\mathrm{m}} \phi\left(-\mathrm{x}_{\mathrm{i}}-\theta\right) \Phi^{\mathrm{i}-1}\left(-\mathrm{x}_{\mathrm{i}}-\theta\right) \phi\left(-\mathrm{y}_{\mathrm{i}}-\theta\right)\left[1-\Phi\left(-\mathrm{y}_{\mathrm{i}}-\theta\right)\right]^{\mathrm{i}-1} \mathrm{~d} \theta}{\int \prod_{\theta}^{\mathrm{m}} \phi\left(-\mathrm{x}_{\mathrm{i}}-\theta\right) \Phi^{\mathrm{i}-1}\left(-\mathrm{x}_{\mathrm{i}}-\theta\right) \phi\left(-\mathrm{y}_{\mathrm{i}}-\theta\right)\left[1-\Phi\left(-\mathrm{y}_{\mathrm{i}}-\theta\right)\right]^{\mathrm{i}-1} \mathrm{~d} \theta} \\
=\frac{\int_{\theta} \theta \prod_{\mathrm{i}=1}^{\mathrm{m}} \phi\left(\mathrm{x}_{\mathrm{i}}+\theta\right) \Phi^{\mathrm{i}-1}\left(-\left(\mathrm{x}_{\mathrm{i}}+\theta\right)\right) \phi\left(\mathrm{y}_{\mathrm{i}}+\theta\right)\left[1-\Phi\left(-\left(\mathrm{y}_{\mathrm{i}}+\theta\right)\right)\right]^{\mathrm{i}-1} \mathrm{~d} \theta}{\int \prod_{\theta}^{m} \phi\left(\mathrm{x}_{\mathrm{i}}+\theta\right) \Phi^{\mathrm{i}-1}\left(-\left(\mathrm{x}_{\mathrm{i}}+\theta\right)\right) \phi\left(\mathrm{y}_{\mathrm{i}}+\theta\right)\left[1-\Phi\left(-\left(\mathrm{y}_{\mathrm{i}}+\theta\right)\right)\right]^{\mathrm{i}-1} \mathrm{~d} \theta},
\end{gathered}
$$

and since $-\mathrm{x}$ has the same distribution as $\mathrm{y}$, we got

$$
\hat{\theta}_{\mathrm{MERSS}}^{\delta}(-(\mathrm{X}, \mathrm{y}))=-\hat{\theta}_{\mathrm{MERSS}}^{\delta}(\mathrm{x}, \mathrm{y}) \text {. }
$$

3. Since $\phi$ is symmetric around $\theta$, we have

$$
\begin{gathered}
\mathrm{E}\left[\hat{\theta}_{\mathrm{MERSS}}^{\delta}(\mathrm{x}-\theta, \mathrm{y}-\theta)\right]=\mathrm{E}\left[\hat{\theta}_{\mathrm{MERSS}}^{\delta}(-(\mathrm{x}-\theta),-(\mathrm{y}-\theta))\right] \\
=-\mathrm{E}\left[\hat{\theta}_{\mathrm{MERSS}}^{\delta}(\mathrm{x}-\theta, \mathrm{y}-\theta)\right],
\end{gathered}
$$

and thus

$$
\mathrm{E}\left[\hat{\theta}_{\mathrm{MERSS}}^{\delta}(\mathrm{X}-\theta, \mathrm{y}-\theta)\right]=0 .
$$


Said Ali Al-Hadhrami, Amer Ibrahim Al-Omari

From (1), we have

$$
\mathrm{E}\left[\hat{\theta}_{\mathrm{MERSS}}^{\delta}(\mathrm{X}, \mathrm{y})\right]=\theta+\mathrm{E}\left[\hat{\theta}_{\mathrm{MERSS}}^{\delta}(\mathrm{X}-\theta, \mathrm{y}-\theta)\right]=\theta \text {. }
$$

4. Follows from (1).

3.2 Bayes estimator of the mean of normal distribution with conjugate prior Now, if $\mathrm{X}_{1}, \mathrm{X}_{2}, \mathrm{~L}, \mathrm{X}_{\mathrm{m}}$ is a SRS from the normal distribution $\mathrm{N}(\theta, 1)$, then the Bayes estimator using $\mathrm{N}(0,1)$ as a prior distribution for $\theta$ is $\hat{\theta}_{\text {SRS }}=\mathrm{m} \bar{X} /(1+\mathrm{m})$, with Bayes risk $=1 /(m+1)$. Also, the Bayes estimator $\hat{\theta}_{S R S}^{\delta}$ based on constant prioris $\bar{X}$ with risk function $1 / \mathrm{m}$.

If $\bar{X}^{*}$ is the average of SRS of size $m(m+1)$ (full data), and $\hat{\theta}_{\mathrm{SRS}}\left(\mathrm{x}^{*}\right)$ is the Bayes estimator for $\theta$ based on the full data, then, from Formula (2) we have

$$
\begin{array}{r}
\hat{\theta}_{\text {MERSS }}(x, y)=E\left(\hat{\theta}_{S R S}\left(x^{*}\right) \mid x, y\right) \\
=\frac{m^{2}+m}{m^{2}+m+1} E\left(\bar{x}^{*} \mid x, y\right) \\
=\frac{m^{2}+m}{m^{2}+m+1} E\left(\hat{\theta}_{\text {SRS }}^{\delta} \mid x, y\right) \\
=\frac{m^{2}+m}{m^{2}+m+1} \hat{\theta}_{\text {MERSS }}^{\delta}(x, y) .(9)
\end{array}
$$

Some properties of the Bayes estimator of the mean of normal distribution using conjugate prior are stated in the next lemma.

\section{Lemma 2:}

1. $E\left(\hat{\theta}_{\mathrm{MERSS}}(\mathrm{x}, \mathrm{y})\right)=\theta\left(\frac{\mathrm{m}^{2}+\mathrm{m}}{\mathrm{m}^{2}+\mathrm{m}+1}\right)$.

2. The Bayes risk is given by

$$
r\left(\hat{\theta}_{\text {MERSS }}(\mathrm{x}, \mathrm{y}), \pi\right)=\frac{1}{\left(\mathrm{~m}^{2}+\mathrm{m}+1\right)^{2}}\left[\left(\mathrm{~m}^{2}+\mathrm{m}\right)^{2} \operatorname{Var}\left(\hat{\theta}_{\mathrm{MERSS}}^{\delta}(\mathrm{x}, \mathrm{y}) \mid \theta\right)+1\right],
$$

3. The efficiency $=\frac{r\left(\hat{\theta}_{\text {SRS }}, \pi\right)}{r\left(\hat{\theta}_{\text {MERSS }}, \pi\right)} \geq 1$.

Proof:

1. To prove (1), take the expectation of the both sides of

as

$$
\hat{\theta}_{\text {MERSS }}(x, y)=\left(\frac{m^{2}+m}{m^{2}+m+1}\right) \hat{\theta}_{\text {MERSS }}^{\delta}(x, y)
$$

$$
\mathrm{E}\left(\hat{\theta}_{\mathrm{MERSS}}(\mathrm{x}, \mathrm{y})\right)=\frac{\mathrm{m}^{2}+\mathrm{m}}{\mathrm{m}^{2}+\mathrm{m}+1} \mathrm{E}\left(\hat{\theta}_{\mathrm{MERSS}}^{\delta}(\mathrm{x}, \mathrm{y})\right),
$$


Bayes Estimation of the Mean of Normal Distribution Using Moving Extreme Ranked Set Sampling which by Lemma(1) will be

$$
E\left(\hat{\theta}_{\text {MERSS }}(\mathrm{x}, \mathrm{y})\right)=\theta\left(\frac{\mathrm{m}^{2}+\mathrm{m}}{\mathrm{m}^{2}+\mathrm{m}+1}\right) \text {. }
$$

2. The variance of $\hat{\theta}_{\mathrm{MERSS}}(\mathrm{x}, \mathrm{y})$ is

$$
\operatorname{Var}\left(\hat{\theta}_{\text {MERSS }}(\mathrm{x}, \mathrm{y})\right)=\left(\frac{\mathrm{m}^{2}+\mathrm{m}}{\mathrm{m}^{2}+\mathrm{m}+1}\right)^{2} \operatorname{Var}\left(\hat{\theta}_{\mathrm{MERSS}}^{\delta}(\mathrm{x}, \mathrm{y})\right),
$$

which is free of $\theta$ by Lemma (1).

Hence,

$$
\begin{aligned}
\mathrm{R}\left(\hat{\theta}_{\text {MERSS }}(\mathrm{x}, \mathrm{y}), \theta\right) & =\operatorname{Var}\left(\hat{\theta}_{\text {MERSS }}(\mathrm{x}, \mathrm{y}) \mid \theta\right)+(\mathrm{biaS})^{2} \\
= & \left(\frac{\mathrm{m}^{2}+\mathrm{m}}{\mathrm{m}^{2}+\mathrm{m}+1}\right)^{2} \operatorname{Var}\left(\hat{\theta}_{\text {MERSS }}^{\delta}(\mathrm{x}, \mathrm{y})\right)+\frac{\theta^{2}}{\left(\mathrm{~m}^{2}+\mathrm{m}+1\right)^{2}},
\end{aligned}
$$

and Bayes risk for $\hat{\theta}_{\text {MERSS }}$ is

$$
\begin{aligned}
r\left(\hat{\theta}_{\text {MERSS }}(\mathrm{x}, \mathrm{y}), \pi\right) & =\mathrm{E}_{\theta}\left[\mathrm{R}\left(\hat{\theta}_{\mathrm{MERSS}}(\mathrm{x}, \mathrm{y}), \theta\right)\right] \\
& =\frac{1}{\left(\mathrm{~m}^{2}+\mathrm{m}+1\right)^{2}}\left(\left(\mathrm{~m}^{2}+\mathrm{m}\right)^{2} \operatorname{Var}\left(\hat{\theta}_{\mathrm{MERSS}}^{\delta}(\mathrm{x}, \mathrm{y}) \mid \theta\right)+1\right) .
\end{aligned}
$$

3. Since the Bayes risk of $\hat{\theta}_{\mathrm{SRS}}$ with size a sample of size $2 \mathrm{mis}$ $r\left(\hat{\theta}_{S R S}, \pi\right)=\frac{1}{2 m+1}$, the efficiency of $\hat{\theta}_{\mathrm{MERSS}}$ with respect to $\hat{\theta}_{\mathrm{SRS}}$ is eff $=\frac{r\left(\hat{\theta}_{\text {SRS }}, \pi\right)}{r\left(\hat{\theta}_{\text {MERSS }}, \pi\right)}$.

The Bayes estimator has the smallest Bayes risk among all other estimators, $r\left(\hat{\theta}_{\mathrm{MERSS}}, \pi\right) \leq \mathrm{r}(\hat{\theta}, \pi)$. Now, let $\hat{\theta}=\frac{2 \mathrm{mw}}{2 \mathrm{~m}+1}$, where $\mathrm{w}$ and $\overline{\mathrm{X}}^{*}$ are the averages based on 2 m units using MERSS and SRS, respectively. Thus,

$$
\begin{aligned}
& r\left(\hat{\theta}_{\text {MERSS }}, \pi\right) \leq r\left(\frac{2 m w}{2 m+1}, \pi\right) \\
& =\mathrm{E}_{\pi}\left(\operatorname{Var}\left(\frac{2 \mathrm{mw}}{2 \mathrm{~m}+1} \mid \theta\right)+(\text { bias })^{2}\right) \\
& =\left(\frac{2 \mathrm{~m}}{2 \mathrm{~m}+1}\right)^{2} \operatorname{Var}(\mathrm{w} \mid \theta)+\frac{1}{(2 \mathrm{~m}+1)^{2}} \\
& \leq \frac{1}{(2 \mathrm{~m}+1)^{2}}\left(4 \mathrm{~m}^{2} \operatorname{Var}\left(\overline{\mathrm{x}}^{*} \mid \theta\right)+1\right) \\
& =\frac{1}{2 \mathrm{~m}+1} .
\end{aligned}
$$


Thus, $r\left(\hat{\theta}_{\mathrm{MERSS}}, \pi\right) \leq \frac{1}{2 \mathrm{~m}+1}$. Since $\operatorname{Var}(\mathrm{w} \mid \theta) \leq \operatorname{Var}\left(\overline{\mathrm{x}}^{*} \mid \theta\right)$ by Al-Odat and Al-Saleh (2000). Therefore,

$$
\text { eff }=\frac{r\left(\hat{\theta}_{\text {SRS }}, \pi\right)}{r\left(\hat{\theta}_{\text {MERSS }}, \pi\right)}=\frac{1 /(2 m+1)}{r\left(\hat{\theta}_{\text {MERSS }}, \pi\right)} \geq 1 .
$$

\section{Approximation of $\hat{\theta}_{\text {MERSS }}$}

From Section (3), we got the Bayes estimator of the mean of normal distribution in the form

$$
\hat{\theta}=\frac{\int_{-\infty}^{\infty} \theta \mathrm{h}(\theta) \prod_{\mathrm{i}=1}^{\mathrm{m}} \mathrm{i}^{2} \phi\left(\mathrm{x}_{\mathrm{i}}-\theta\right)\left[\Phi\left(\mathrm{x}_{\mathrm{i}}-\theta\right)\right]^{\mathrm{i}-1} \phi\left(\mathrm{y}_{\mathrm{i}}-\theta\right)\left[1-\Phi\left(\mathrm{y}_{\mathrm{i}}-\theta\right)\right]^{\mathrm{i}-1} \mathrm{~d} \theta}{\int_{-\infty}^{\infty} \mathrm{h}(\theta) \prod_{\mathrm{i}=1}^{m} \mathrm{i}^{2} \phi\left(\mathrm{x}_{\mathrm{i}}-\theta\right)\left[\Phi\left(\mathrm{x}_{\mathrm{i}}-\theta\right)\right]^{\mathrm{i}-1} \phi\left(\mathrm{y}_{\mathrm{i}}-\theta\right)\left[1-\Phi\left(\mathrm{y}_{\mathrm{i}}-\theta\right)\right]^{\mathrm{i}-1} \mathrm{~d} \theta} .
$$

This estimator is very complicated. However, it can be approximated using numerical methods (see Ross 1997). One can use Monte Carlo method of approximation. That is, if it is possible to generate random variables $\theta_{1}, \theta_{2}, \ldots, \theta_{\mathrm{r}}$ from $h(\theta)$, where $r$ goes to $+\infty$, then we can approximate the Bayes estimator by

$$
\hat{\theta}_{1}=\frac{\sum_{\mathrm{t}=1}^{\mathrm{r}} \theta_{\mathrm{t}} \prod_{\mathrm{i}=1}^{\mathrm{m}} \mathrm{i}^{2} \phi\left(\mathrm{x}_{\mathrm{i}}-\theta_{\mathrm{t}}\right)\left[\Phi\left(\mathrm{x}_{\mathrm{i}}-\theta_{\mathrm{t}}\right)\right]^{\mathrm{i}-1} \phi\left(\mathrm{y}_{\mathrm{i}}-\theta_{\mathrm{t}}\right)\left[1-\Phi\left(\mathrm{y}_{\mathrm{i}}-\theta_{\mathrm{t}}\right)\right]^{\mathrm{i}-1}}{\sum_{\mathrm{t}=1}^{\mathrm{r}} \prod_{\mathrm{i}=1}^{\mathrm{m}} \mathrm{i}^{2} \phi\left(\mathrm{x}_{\mathrm{i}}-\theta_{\mathrm{t}}\right)\left[\Phi\left(\mathrm{x}_{\mathrm{i}}-\theta_{\mathrm{t}}\right)\right]^{\mathrm{i}-1} \phi\left(\mathrm{y}_{\mathrm{i}}-\theta_{\mathrm{t}}\right)\left[1-\Phi\left(\mathrm{y}_{\mathrm{i}}-\theta_{\mathrm{t}}\right)\right]^{\mathrm{i}-1}},
$$

\section{Simulation Study}

In order to evaluate the behavior of the Bayes estimator, a simulation was conducted. Some values of $\theta$ was generated from $N(0,1)$ and samples are selected from $N(\theta, 1)$ using MERSS. Then, $\hat{\theta}_{1}$ was calculated from the sample and the risk function was obtained and compared with that from simple random sample. The results are reported in Table 1.

Table 1: Simulation results for comparison between Bayes risks of the estimators using MERSS and SRS $m=3,5,7,10,15$.

\begin{tabular}{|c|c|c|c|}
\hline $\mathrm{m}$ & $\mathrm{R}_{\text {MERSS }}$ & $\mathrm{R}_{\text {SRS }}$ & Efficiency \\
\hline 3 & 0.1122 & 0.1461 & 1.30 \\
\hline 5 & 0.0596 & 0.0913 & 1.53 \\
\hline 7 & 0.0384 & 0.0660 & 1.72 \\
\hline 10 & 0.0241 & 0.0478 & 1.99 \\
\hline 15 & 0.0141 & 0.0322 & 2.28 \\
\hline
\end{tabular}


Bayes Estimation of the Mean of Normal Distribution Using Moving Extreme Ranked Set Sampling

Based on Table 1, the efficiency is greater than 1 for the set sizes considered in this study and is increasing with $\mathrm{m}$. This indicates that MERSS estimator is more efficient than SRS estimator when estimating the mean of normal distribution.

\section{Conclusions}

The Bayes estimation of the mean of normal distribution using MERSS is considered and compared with the estimators based SRS. It is recommended to use MERSS for estimating the population mean of the normal distribution using Bayes estimation.

\section{References}

1. Abu-Dayyeh, W. and Al-Sawi, E. (2007). Modified inference about the mean of the exponential distribution using moving extreme ranked set sampling. Statistical Papers, 50(2): 249-259.

2. Al-Hadhrami, S.A. (2007). Estimation of the population mean using ranked set sampling with auxiliary variable. PhD Thesis, UKM, Malaysia.

3. Al-Hadhrami, S.A. and Al-Omari, A.I. (2010). Bayesian inference on the variance of normal distribution using moving extreme ranked set sampling. J ournal of Modern Applied Statistical Methods, 8(1), 273-281.

4. Al-Hadhrami, S.A., Al Omari, A.E. and Al-Saleh, M.F. (2009). Estimation of standard deviation of normal distribution using moving extreme ranked set sampling. World Academy of Science, Engineering and Technology. 37, 988-993.

5. Al-Odat, M.T. and Al-Saleh, M.F. (2001).A variation of ranked set sampling. J ournal of Applied Statistical Science, 10, 137-146.

6. Al-Omari, A.I., Ibrahim, K., Jemain, A.A. and Al-Hadhrami, S.A. (2009). Multistage balanced groups ranked set samples for estimating the population median, Statistics in Transition, 10(2), 223-233.

7. Al-Omari, A.I. and Jaber, K. (2008). Percentile double ranked set sampling. J ournal of Mathematics and Statistics, 4(1), 60-64.

8. Al-Saleh, M.F. and Al-Ananbeh, A.M. (2007). Estimation of the means of the bivariate normal using moving extreme ranked set sampling with concomitant variable. Statistical Papers, 48(2): 179-195.

9. Al-Saleh, M.F. and Abu Hawwas J. (2002).Characterization of ranked set sampling Bayes estimators with application to the normal distribution. Soochow J ournal of Mathematics, 28, 223-234.

10. Al-Saleh, M.F. and Al-Hadhrami, S. (2003a).Parametric estimation for the location parameter for symmetric distributions using moving extremes ranked set sampling with application to trees data. Environmetrics, 14(7), 651-664.

11. Al-Saleh, M.F. and Al-Hadhrami, S. (2003b). Estimation of the mean of exponential distribution using moving extreme ranked set sampling. Statistical Papers, 44, 367-387. 
12. Al-Saleh, M.F., Al-Sharfat. K. and Muttlak, H. (2000). Bayesian estimation using ranked set sampling. Biometrical J ournal, 42, 1-12.

13. Al-Saleh, M.F. and Samawi, H. (2010).On estimating the odds using moving extreme ranked set sampling. Statistical Methodology, 7 (2), 133- 140.

14. Al-Saleh, M.F. and Muttlak, H. (1998). A note on the estimation of the parameter of the exponential distribution using Bayesian RSS. Pakistan J ournal of S tatistics, 14, 49-56.

15. Kim and Arnold (1999). Parameter estimation under generalized ranked set sampling. Statistics and Probability Letters, 42, 353-360.

16. Koyuncu, N. and Kadilar, C. (2010). Ratio and product estimators in stratified random sampling. Journal of Statistical Planning and Inference, 139, 2552-2558.

17. Lavine, M. (1999). The Bayesics of ranked set sampling. J ournal of Environmental and E cological S tatistics, 6, 47-57.

18. LI, T. and Balakrishnan, N. (2008). Best linear unbiased estimators of parameters of a simple linear regression model based on ordered ranked set samples. J ournal of Statistical Computation and Simulation, 78(12), 1267-1278.

19. Rubin, D. (1987). Multiple imputations for nonresponse in sample surveys and censuses. Wiley, New York.

20. Sheldom, M. Ross (1997). Simulation, 2nd Edition, Academic Press, USA. 